\title{
Type 1 diabetic patients with peripheral neuropathy have pan-enteric prolongation of gastrointestinal transit times and an altered caecal $\mathrm{pH}$ profile
}

\author{
Adam D. Farmer 1,2,3 - Anne Grave Pedersen ${ }^{3,4}$ • Birgitte Brock B $^{4,5}$ \\ Poul Erik Jakobsen $^{6}$ • Jesper Karmisholt ${ }^{6}$ - Sahar D. Mohammed ${ }^{2}$. \\ S. Mark Scott ${ }^{2}$ - Asbjørn Mohr Drewes ${ }^{3}$ - Christina Brock ${ }^{3,5,7}$
}

Received: 17 September 2016/Accepted: 7 December 2016/Published online: 20 January 2017

(C) Springer-Verlag Berlin Heidelberg 2017

\begin{abstract}
Aims/hypothesis We hypothesised that type 1 diabetic patients with established diabetic sensorimotor polyneuropathy (DSPN) would have segmental and/or pan-enteric dysmotility in comparison to healthy age-matched controls. We aimed to investigate the co-relationships between gastrointestinal function, degree of DSPN and clinical symptoms.

Methods An observational comparison was made between 48 patients with DSPN (39 men, mean age 50 years, range 29-71 years), representing the baseline data of an ongoing clinical trial (representing a secondary analysis of
\end{abstract}

Electronic supplementary material The online version of this article (doi:10.1007/s00125-016-4199-6) contains peer-reviewed but unedited supplementary material, which is available to authorised users.

Christina Brock

christina.brock@rn.dk

1 Department of Gastroenterology, University Hospitals of North Midlands, Stoke on Trent, UK

2 Centre for Neuroscience and Trauma, Blizard Institute, Wingate Institute of Neurogastroenterology, Barts and the London School of Medicine and Dentistry, Queen Mary University of London, London, UK

3 Mech-Sense, Department of Gastroenterology and Hepatology, Aalborg University Hospital, Mølleparkvej 4, DK-9000 Aalborg, Denmark

4 Clinical Biochemistry, Aarhus University Hospital, Aarhus, Denmark

5 Department of Clinical Medicine, Aarhus University, Aarhus, Denmark

6 Department of Endocrinology, Aalborg University Hospital, Aalborg, Denmark

7 Department of Drug Design and Pharmacology, University of Copenhagen, Copenhagen, Denmark baseline data collected from an ongoing double-blind randomised controlled trial investigating the neuroprotective effects of liraglutide) and 41 healthy participants (16 men, mean age 49 years, range 30-78) who underwent a standardised wireless motility capsule test to assess gastrointestinal transit. In patients, vibration thresholds, the Michigan Neuropathy Screening Instrument and Patient Assessment of Upper Gastrointestinal Symptom questionnaires were recorded.

Results Compared with healthy controls, patients showed prolonged gastric emptying $(299 \pm 289$ vs $179 \pm 49 \mathrm{~min}$; $p=0.01)$, small bowel transit $(289 \pm 107$ vs $224 \pm 63 \mathrm{~min}$; $p=0.001)$, colonic transit $(2140$, interquartile range [IQR] 1149-2799 min vs 1087, IQR 882-1650 min; $p=0.0001$ ) and whole-gut transit time (2721, IQR 1196-3541 min vs 1475 (IQR 1278-2214) min; $p<0.0001$ ). Patients also showed an increased fall in $\mathrm{pH}$ across the ileocaecal junction $(-1.8 \pm 0.4$ vs $-1.3 \pm 0.4 \mathrm{pH} ; p<0.0001)$, which was associated with prolonged colonic transit $(r=0.3, p=0.001)$. Multivariable regression, controlling for sex, disease duration and glycaemic control, demonstrated an association between whole-gut transit time and total GCSI $(p=0.02)$.

Conclusions/interpretation Pan-enteric prolongation of gastrointestinal transit times and a more acidic caecal $\mathrm{pH}$, which may represent heightened caecal fermentation, are present in patients with type 1 diabetes. The potential implication of delayed gastrointestinal transit on the bioavailability of nutrition and on pharmacotherapeutic and glycaemic control warrants further investigation.

Trial registration EUDRA CT: 2013-004375-12

Keywords Caecal fermentation - Diabetes mellitus · Gastrointestinal dysfunction - Gastrointestinal transit • Pan-enteric dysfunction · SmartPill 


$\begin{array}{ll}\text { Abbreviations } \\ \text { CTT } & \text { Colonic transit time } \\ \text { DAN } & \text { Diabetic autonomic neuropathy } \\ \text { DSPN } & \text { Diabetic sensorimotor polyneuropathy } \\ \text { GCSI } & \text { Gastroparesis Cardinal Symptom Index } \\ \text { GET } & \text { Gastric emptying time } \\ \text { HRV } & \text { Heart rate variability } \\ \text { ICJ } & \text { Ileocaecal junction } \\ \text { IQR } & \text { Interquartile range } \\ \text { MNSI } & \text { Michigan Neuropathy Screening Instrument } \\ \text { PAGI-SYM } & \text { Patients Assessment of Upper } \\ & \text { Gastrointestinal Symptoms } \\ \text { SBTT } & \text { Small bowel transit time } \\ \text { SIBO } & \text { Small intestinal bacterial overgrowth } \\ \text { WGTT } & \text { Whole-gut transit time } \\ \text { WMC } & \text { Wireless motility capsule }\end{array}$

\section{Introduction}

Type 1 diabetes is associated with microvascular disturbances leading to neuropathy in up to $50 \%$ of patients [1]. Diabetic peripheral neuropathy consists of two separate entities - diabetic sensorimotor polyneuropathy (DSPN) and diabetic autonomic neuropathy (DAN) [1] - which may or may not coexist in individual patients [2]. Classically, DSPN affects sensory and motor neurons in a 'stocking and glove' pattern, whereas DAN affects the cardiovascular, urogenital, sudomotor and gastrointestinal systems [1,3]. Within the gastrointestinal tract, this may lead to sensorimotor aberrations, and symptoms such as nausea, vomiting, bloating, early satiety, distension and abdominal pain are frequently encountered [4-7].

Although the most frequently identified gastrointestinal complication of diabetes is gastroparesis [8], which can itself exert a negative impact on the patient's glycaemic control and quality of life, gastrointestinal manifestations remain underrecognised [9]. Over a 10-year period, the cumulative HR for developing gastroparesis, relative to healthy controls, is 33 (95\% CI 2,274) in patients with type 1 diabetes in contrast to $7.5(95 \%$ CI $0.8,68)$ in those with type 2 diabetes [10]. Evidence suggests that the societal, economic and healthcare burden of gastroparesis is rising, arguably as a consequence of heightened clinical suspicion and improved access to diagnostics [11]. Given that gastroparesis represents a diminution in foregut motility, it can result in an unpredictable delivery of nutrients and medication from the stomach into the small bowel, which can adversely affect nutritional status, efficacy of medical treatment and glycaemic control [12]. Considering that the gastrointestinal tract is a continuous organ from mouth to anus, it is plausible to suggest that gastrointestinal dysmotility in type 1 diabetes is not purely confined to the foregut and that symptoms originating in one part of the gut may overlap with those in another. Localisation of any dysfunction is now recognised as a crucial first step for accurate diagnosis and further management $[13,14]$.

Contemporaneous assessment of motility throughout the gastrointestinal tract generally utilises regional or pan-enteric scintigraphic evaluation. Such methods are, however, timeconsuming and necessitate exposure to radiation. The wireless motility capsule (WMC) is a novel validated method that measures regional and whole-gut transit times (WGTTs) in a noninvasive ambulatory manner within a single test [15]. The WMC consists of an indigestible single-use polyurethane capsule that measures pressure, $\mathrm{pH}$ and temperature as it traverses the gastrointestinal tract. Regional transit times are derived from stereotypical changes in gut $\mathrm{pH}$. The magnitude of change in $\mathrm{pH}$ around the ileocaecal junction (ICJ) has recently been proposed as an objective surrogate biomarker of caecal fermentation, reflecting changes in the microbiota $[16,17]$. We therefore hypothesised that a proportion of patients with type 1 diabetes and proven DSPN have gastrointestinal dysmotility, and we questioned whether any dysmotility identified would be regional or pan-enteric. We aimed to describe any differences between patients and healthy controls in terms of both regional and total transit times and ileocaecal $\mathrm{pH}$ patterns. Furthermore, we wanted to investigate the relationship between these findings and self-reported gastrointestinal symptoms and quality of life, as well as objective markers of DSPN.

\section{Methods}

\section{Study population}

The study comprised an observational comparison involving 48 consecutive patients with type 1 diabetes and verified DSPN (39 men, mean age 50 years, range 29-71 years), representing a secondary analysis of baseline data collected from an ongoing double-blind randomised controlled trial investigating the neuroprotective effects of liraglutide (Treatment of Diabetic Neuropathy With Liraglutide [TODINELI] trial; EUDRA CT 2013-004375-12). Patients were recruited at the Department of Endocrinology, Aalborg University Hospital, Denmark. Potential eligible patients were pre-screened on the basis of a recorded vibration perception threshold of over $18 \mathrm{~V}$, and DSPN was verified by nerve conduction tests. No patients had a history of osteoporosis. Additional inclusion criteria were age over 18 years and a verified diagnosis of type 1 diabetes for a minimum of 2 years $\left(\mathrm{HbA}_{1 \mathrm{c}} \geq 6.5 \%\right.$ [ $\left.\left.>48 \mathrm{mmol} / \mathrm{mol}\right]\right)$. Patients' characteristics are listed in Table 1 . These patients were compared with 41 age-matched healthy participants from an established normal dataset (16 men, mean age 49 years, range $30-78$ years) [15]. All healthy participants had a normal bowel habit, defined as between three bowel movements per day and one bowel movement every 3 days, with no 
Table 1 Demographic differences between patients, including their clinical data, and healthy participants

\begin{tabular}{|c|c|c|c|}
\hline & Patients & Healthy participants & $p$ value \\
\hline Sex & 39 men, 9 women & 25 men, 16 women & 0.06 \\
\hline Age (range), years & $500(29-71)$ & $49(30-78)$ & 0.89 \\
\hline $\begin{array}{l}\text { Duration of diabetes (range), years } \\
\text { BMI, } \mathrm{kg} / \mathrm{m}^{2}\end{array}$ & $\begin{array}{l}32(14-51) \\
28.5 \pm 4.9\end{array}$ & & \\
\hline Weight, kg & $90.1 \pm 16.0$ & & \\
\hline Weight gain in the preceding $8 \pm 4$ months, $\mathrm{kg}$ & $1.4 \pm 4.2$ & & \\
\hline Heart rate, beats/min & $73.9 \pm 10.5$ & & \\
\hline Systolic BP, mmHg & $149.9 \pm 16.5$ & & \\
\hline Diastolic BP, mmHg & $82.3 \pm 10.9$ & & \\
\hline Mean $\mathrm{R}-\mathrm{R}$ interval, $\mathrm{ms}^{\mathrm{a}}$ & $775.6 \pm 105.5$ & & \\
\hline SDNN & $114.1 \pm 34.8$ & & \\
\hline SDNNi & $41.4 \pm 18.3$ & & \\
\hline SDANN & $104.7 \pm 31.9$ & & \\
\hline RMSSD & $19.3 \pm 9.6$ & & \\
\hline $\mathrm{HbA}_{1 \mathrm{c}}, \mathrm{mmol} / \mathrm{mol}$ & $65.5 \pm 9.7$ & & \\
\hline $\mathrm{HbA}_{1 \mathrm{c}}, \%$ & $8.1 \pm 1$ & & \\
\hline Serum creatinine, $\mu \mathrm{mol} / 1$ & $84.0 \pm 20.1$ & & \\
\hline Estimated GFR, $\mathrm{ml} \mathrm{min}^{-1}[1.73 \mathrm{~m}]^{-2}$ & $78.9 \pm 12.8$ & & \\
\hline Serum calcium $(n=40), \mathrm{mmol} / \mathrm{l}^{\mathrm{b}}$ & $2.35 \pm 0.1$ & & \\
\hline No. of patients with insulin pumps (M/F) & $10 / 48$ & & \\
\hline Use of rapid-acting insulin $(n=38)$, IU/day & $35 \pm 19$ & & \\
\hline Use of long-acting insulin $(n=38)$, IU/day & $34 \pm 22$ & & \\
\hline No. taking antihypertensives $(\mathrm{M} / \mathrm{F})$ & $33 / 48$ & & \\
\hline No. taking antihypercholesterolaemic agents $(\mathrm{M} / \mathrm{F})$ & $24 / 48$ & & \\
\hline
\end{tabular}

Data are expressed as mean and SD unless otherwise stated

${ }^{\mathrm{a}} \mathrm{R}-\mathrm{R}$ interval: the time between successive $\mathrm{R}$ waves on the ECG

${ }^{\mathrm{b}}$ Calcium levels were not part of the study protocol; therefore these values represent the most recent values in the medical record

RMSSD, root mean square differences of successive R-R intervals; SDANN, SD of sequential 5 min R-R interval means; SDNN, SD of normal-to-normal R-R intervals; SDNNi, mean of SDs of all normal-to-normal intervals in all 5 min segments of the ECG recording gastrointestinal symptoms that were particularly suggestive of either small intestinal bacterial overgrowth (SIBO) or a rectal evacuatory disorder. No healthy participants had any gastrointestinal symptoms or history of metabolic, neurogenic or endocrine disorder known to influence gastrointestinal motor activity. In addition, no participant had undergone gastrointestinal surgery, other than appendectomy and/or cholecystectomy, and none was taking either laxatives or medications known to influence gastrointestinal motility or $\mathrm{pH}$.

\section{Protocol}

Prior to entry into the study, all participants gave written informed consent. Patients underwent testing of peripheral nerve and autonomic nervous system function and completed a number of validated questionnaires prior to ingesting a WMC. Serum blood glucose concentrations were measured directly before participants ingested the WMC. Healthy participants only underwent WMC investigation and had a full history and clinical examination performed to confirm the absence of any exclusion criteria. All patient protocols were approved by the Ethics Committee for North Jutland, Denmark (EUDRA CT 2013-004375-12, Local ethical committee N-20130077).

\section{Peripheral nerve function testing}

Vibration perception threshold The threshold for vibratory perception was measured using a biothesiometer (BioMedical Instruments, Newbury, OH, USA), with the amplitude of the vibrations indicated by a voltmeter. The highest recordable value was $50 \mathrm{~V}$. The vibrator was hand-held and applied to the dorsum of the first phalanx of each foot. The stimulus strength was increased gradually from zero to first sensation, and the test was repeated twice on both feet. The average was derived as the vibration perception threshold on each foot. 
Peripheral nerve conduction test The median, ulnar, sural, radial, tibial and peroneal nerves were evaluated, including sensory and motor components. However, for this study, only the results for sensory median nerve testing are included. To avoid temperature influencing the conduction velocity, appropriate warming measures were used to ensure that skin temperatures were at least $32^{\circ} \mathrm{C}$. Spring-ring electrodes were used for recording digital sensory nerve action potentials. For all other nerves, a plastic bar electrode was used.

\section{Heart rate variability}

All patients undertook a 24-h ECG recording from which heart rate variability (HRV) indices were derived (for further details, see the electronic supplementary material [ESM]).

\section{Questionnaires}

Michigan Neuropathy Screening Instrument The validated Michigan Neuropathy Screening Instrument (MNSI) was administered to screen for DSPN. A score of $>7$ is considered abnormal.

PAGI-SYM questionnaire Gastrointestinal symptoms in the preceding 2 weeks were assessed using the Patient Assessment of Gastrointestinal Disorder Severity (PAGI-SYM), from which the Gastroparesis Cardinal Symptom Index (GCSI) was derived. (See ESM Table 1 for further details.)

\section{Wireless motility capsule}

The WMC (SmartPill, Medtronic, Minneapolis, MN, USA) consists of a wireless-transmitting, single-use capsule, a portable receiver and display software [18]. (See the ESM for further details.) The regional transit times derived from the electronic data were defined as follows according to the method of Sarosiek et al [19].

Time of capsule ingestion was identified by an abrupt rise in the recorded temperature and a fall in $\mathrm{pH}$ (reflecting passage into the acidic environment of the stomach) (Fig. 1) [15].

Gastric emptying time (GET) was defined as the time between WMC ingestion and the abrupt increase in $\mathrm{pH}(>2 \mathrm{pH}$ units) caused when the capsule moved through the pylorus from the acidic stomach to the more neutral/alkaline environment of the duodenum.

Small bowel transit time (SBTT) was defined as the time between the end of GET and the passage of the WMC through the ICJ. The ICJ was localised by identifying a sudden fall in $\mathrm{pH}(>1 \mathrm{pH}$ unit) that followed a gradual and sustained rise in $\mathrm{pH}$ through the terminal ileum ( $\mathrm{pH}$ typically $7-8$ ).

Colonic transit time (CTT) was defined as the time between entry of the WMC into the caecum, defined from the $\mathrm{pH}$ drop across the ICJ, to its exit from the body. This was determined in one of two ways: (1) by observing an abrupt fall in temperature followed by a loss of recorded signal after the individual had expelled the WMC, or (2) termination of the datarecording coinciding with a bowel movement recorded in the participant's diary.

WGTT was calculated as the time between capsule ingestion and capsule expulsion.

\section{Study endpoints}

The primary endpoint was WGTT. The secondary endpoints were the regional transit times of GET, SBTT and CTT, the magnitude of the $\mathrm{pH}$ change across the ICJ and the association of symptoms with questionnaire scores.

\section{Statistical analysis}

Data distribution was evaluated using visual inspection of histograms and Shapiro-Wilk testing. Quantitative data are presented either as median with interquartile ranges (IQRs) for non-normally distributed data, or mean $\pm \mathrm{SD}$ for normally distributed data. For quantitative data, differences between the groups were assessed using the unpaired $t$ test for parametric data and the Mann-Whitney $U$-test test for non-parametric data. Categorical data were compared using Fisher's exact test. Spearman and Pearson coefficients ( $r_{\mathrm{s}}$ and $r$, respectively) are reported dependent on data distribution. Multivariable linear regression was undertaken to assess the association between the primary outcome and total GCSI, adjusted for prespecified potential confounders of disease duration, sex and glycaemic control. $p$ values of $<0.05$ were adopted as the statistical criterion. Based on previously reported normative data for the primary outcome [15], a sample size of 41 patients and 41 healthy controls had in excess of $80 \%$ power, at a two-sided alpha of 0.05 , to detect a difference of $33 \%$ in WGTT (WGTT $1932 \mathrm{~min} \pm 637 \mathrm{~min}$ ) with an SD of $997 \mathrm{~min}$. The statistical analysis was undertaken using proprietary software (STATA, v.12 Stata Corp LP; College Station, TX, USA).

\section{Results}

All participants completed the study, although the WMC recording in one patient was unavailable for interpretation because of signal loss during the test. There were no age differences between patients and controls (Table 1).

\section{Peripheral nerve function}

Nerve conduction testing and vibration perception thresholds were obtained for all patients. The main outcome of the nerve conduction tests was the sensory nerve action potential of the 


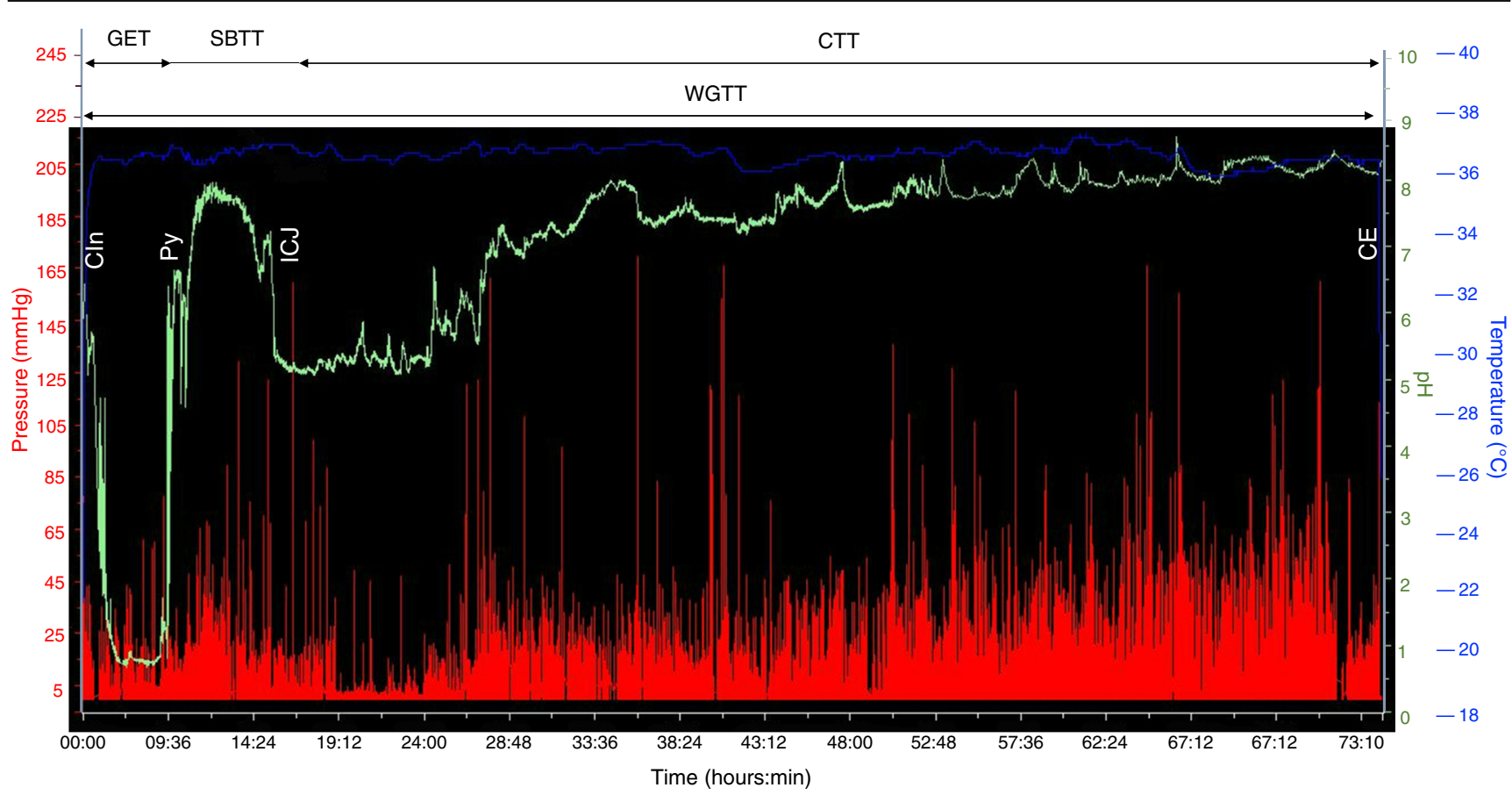

Fig. 1 A typical WMC trace demonstrating temperature (blue line), $\mathrm{pH}$ (green line) and contractility (red line). GET is defined as the time from capsule ingestion (CIn) to a sharp rise in $\mathrm{pH}$ at the pylorus (Py). SBTT is defined as the time between the reading at the pylorus and the abrupt fall

median nerve. In comparison to known reference values from healthy participants [20], the amplitude of the sensory nerve action potential in patients was decreased (median $11 \mu \mathrm{V}$, IQR $8-16 \mu \mathrm{V})$.

\section{Heart rate variability}

All HRV variables were within normal age-specific ranges [21].

\section{Gastrointestinal transit}

In comparison to healthy controls, patients had a significantly prolonged WGTT, GET, SBTT and CTT (Fig. 2, ESM Table 2). There was a positive association between vibration perception thresholds and GET and SBTT $(r=0.33, p=0.02$ and $r=0.38$, $p=0.01$, respectively), but not WGTT. The mean baseline blood glucose was $8.4 \mathrm{mmol} / \mathrm{l}$ (range $4.9-12.1 \mathrm{mmol} / \mathrm{l}$ ). There were no associations between blood glucose concentrations or sensory nerve action potentials and segmental/total transit times. In comparison to published reference values [15], 21/48 patients (44\%) had a single, multisegmental or pan-segmental pathological delay in transit times (see Fig. 3).

\section{Gastrointestinal pH profile}

In comparison to healthy controls, the magnitude of the $\mathrm{pH}$ drop across the ICJ was significantly higher among patients than controls $(-1.8 \pm 0.4$ vs $-1.3 \pm 0.4 ; p<0.0001)$. In in $\mathrm{pH}$ at the ICJ. CTT is defined as the time between the ICJ reading and capsule expulsion (CE), denoted by a rapid fall in temperature as the capsule exits the body. WGTT is derived as the time between CI and CE

comparison to published reference values [15], 11/48 patients (23\%) had a pathological elevation of drop in $\mathrm{pH}$ across the ICJ. Furthermore, the fall in ICJ was positively associated with a prolonged CTT $\left(r_{\mathrm{s}}=0.3, p<0.001\right)$.

\section{Questionnaires}

PAGI-SYM was completed by all patients. PAGI-SYM provides the subscale nausea, fullness and bloating, and from these scales the total GCSI can be calculated. The mean GCSI was $0.51 \pm 0.64$ (see ESM Table 2). There was an association between WGTT and GCSI when controlling for disease duration, sex and glycaemic control $\left(R^{2}=0.27, p=0.02\right.$, $95 \%$ CI $145,1251 \mathrm{~min})$.

\section{Discussion}

In this study, we have demonstrated that, in comparison to an age-matched cohort of healthy controls, patients with type 1 diabetes and established DSPN as a group had pan-enteric prolongation of gastrointestinal transit times. In addition, we found a more profound fall in $\mathrm{pH}$ across the ICJ, which may be a surrogate for heightened caecal fermentation, which was associated with a prolonged CTT. These alterations were present in patients without significant gastrointestinal symptoms, although the total GCSI was associated with a prolonged WGTT. To the best of our knowledge, this is the largest study 
Fig. 2 Box and whisker plots demonstrating differences between GET (a), SBTT (b), CTT (c) and WGTT (d) in patients compared with controls. $* * p<0.01, * * * p<0.001$ a

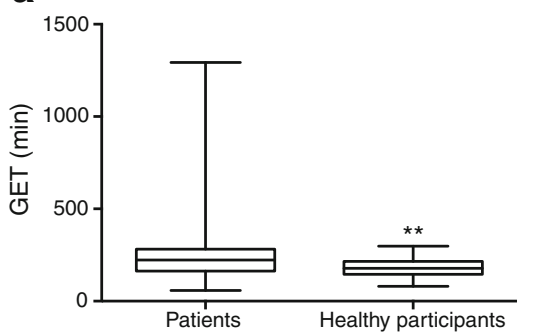

C

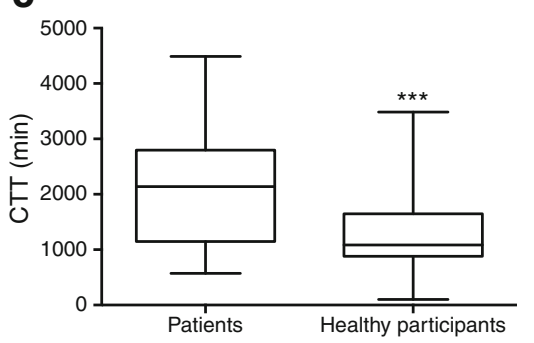

b

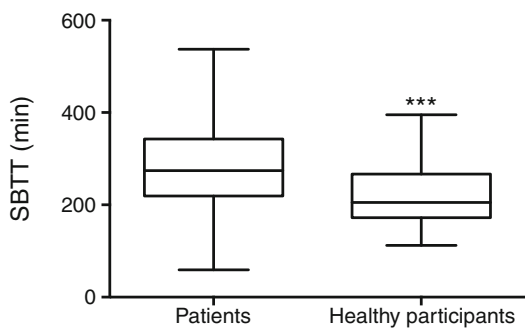

d

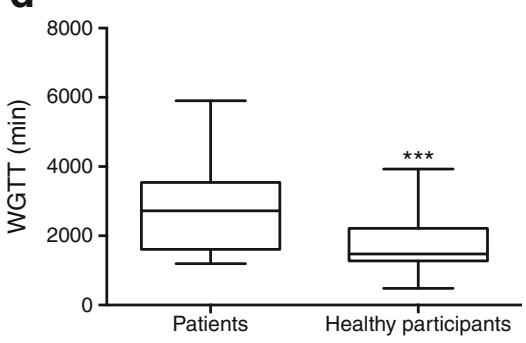

in the literature to date reporting segmental and pan-enteric transit times in patients with type 1 diabetes using a WMC.

Gastrointestinal motility is regulated and coordinated through a complex bidirectional relationship between the autonomic, central and enteric nervous systems, involving various endocrine and hormonal pathways as well as an interplay with gut microbiota. Despite the fact that the pathogenesis of gastrointestinal dysmotility in diabetes is multifactorial, DAN is a pivotal facet [22]. DAN and DSPN are thought to share a common pathophysiology and therefore frequently coexist in the same patient [23]. This observation is supported by the association, albeit moderate, between vibration perception thresholds and regional transit times. In a previous study by Merio et al, no such association was seen between GET and DSPN, which may reflect differences in patient demographics, duration or severity of disease, as well as the variance in methodologies used to assess and classify the degree

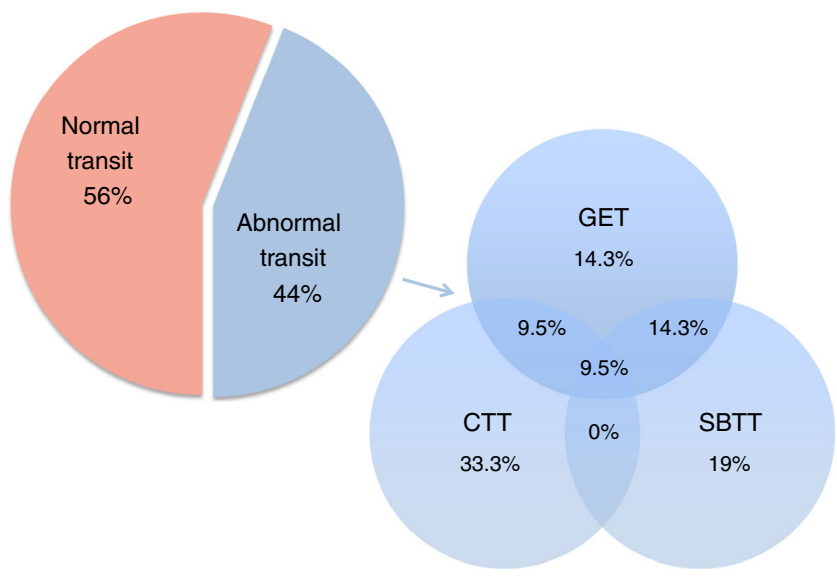

Fig. 3 Venn diagram of the co-relationships in the 21/48 patients (44\%) who had a pathological single, multisegmental or pan-segmental delay in transit times in comparison to published normal values and severity of DSPN [22]. In the current study, we found a prolongation of regional and WGTTs in patients with DSPN, potentially indicating the coexistence of DAN in this group, although there was no objective evidence of DAN, as indexed by HRV variables and renal function, in our patient cohort. Interestingly, the time domain analysis of HRV gave lower values in these patients in comparison to age-matched controls, although this was still within the normal range; this suggests that (at least at the point of time when the study was performed) there was no overt evidence of DAN [21]. Nevertheless, disagreement remains over the absolute utility, as well as the interpretation, of HRV variables in diagnosing DAN. However, DAN is not the only factor in the development of gastrointestinal dysmotility as recent data have provided objective support for the emerging role of the enteric nervous system including, but not limited to, enteric neurons and their interconnections, smooth muscle myopathy, changes in macrophage populations in the muscularis, changes in function of the interstitial cells of Cajal and neurotransmission in the development of diabetic enteropathic syndromes [24, 25].

In agreement with previously reported studies, we found an association between patient-reported symptoms and transit times [26]. Sfarti et al reported that $65 \%$ of 69 patients with type 1 diabetes experienced upper abdominal symptoms, but only bloating and upper abdominal pain were significantly associated with a delayed GET [27]. In contrast, Jones et al demonstrated an association between bloating and postprandial fullness with retarded gastric emptying of solids [28]. Taken together, the majority of studies to date have variably identified certain associations between symptoms and segmental transit times, although concordance between studies is limited. A number of factors could potentially explain this variability. For instance, differences in patient selection (type 1 vs type 2 diabetes, duration of disease, glycaemic control, 
selection bias) and the method by which GET was measured (scintigraphy, breath test, WMC) could account for a proportion of this. Moreover, a plethora of other mechanisms, such as dietary habits, alterations in the microbiota [29], psychological factors and central nervous system abnormalities, might also contribute to the genesis and maintenance of symptoms, rather than these being caused purely by changes in transit times per se [30]. Neuronal reorganisation within the central nervous system has been demonstrated in diabetes, and more specifically it has been shown that gastrointestinal symptoms are associated with deeper localisation of dipolar sources in the insula-operculum region [3]. Moreover, in diabetes patients with gastrointestinal symptoms, alterations in the following brain areas have been demonstrated: (1) brainstem/operculum/frontal cortex, (2) operculum/cingulate, and (3) mid-cingulate/anterior-cingulate/operculum/deep limbic structures [31].

Gastric emptying scintigraphy is currently considered the gold standard for diagnosing gastroparesis [32], although the diagnostic accuracy of the WMC has been demonstrated to be comparable [33, 34]. However, other techniques are available to assess GET, such as transabdominal ultrasonography, MRI, radio-opaque markers, the paracetamol absorption test and breath-testing with ${ }^{13} \mathrm{C}$-octanoic acid. In comparison to gastric emptying scintigraphy and radio-opaque markers, an advantage of the WMC is that it does not involve ionising radiation. Furthermore, the use of both ultrasonography and MRI is limited by the need to purchase specialised equipment as well as by expertise in interpreting the data [35]. Moreover, scintigraphic and breath-testing methods demand a prolonged clinic/office visit of around $6 \mathrm{~h}$. The WMC additionally offers the opportunity to assess the entire gastrointestinal tract, in terms of both segmental and total transit times and $\mathrm{pH}$ patterns, in an ambulatory and relatively minimally invasive manner. However, the absolute relation of the WMC to underlying gastrointestinal motility, due to the indigestible nature of the capsule, coupled with the fact that it is 'free-floating' within the gastrointestinal tract, remains uncertain.

The important clinical implication of our results is the finding of a pan-enteric prolongation of transit times in patients who have hitherto not reported any significant gastrointestinal symptoms, in comparison to healthy control participants. Moreover, nearly half (44\%) of such patients have pathologically delayed segmental and/or whole-gut transit. Although prolongation of CTT and WGTT have been previously reported by Sarosiek et al, their study only involved 15 patients with diabetes already known to have gastroparesis [19]. Taken together, these results suggest that pan-enteric alterations in transit are present in patients with diabetes, and therefore testing strategies should not be confined purely to the foregut. Indeed, our results raise the intriguing question of whether type 1 diabetic patients with DSPN should be actively screened for gastrointestinal dysmotility, even in the absence of bothersome symptoms, as demonstrable dysmotility can cause an unpredictable delivery of nutrients to the small bowel and therefore negatively affect glycaemic control and have a potentially deleteriously impact on long-term outcomes [36].

The patho-aetiology of gastrointestinal dysmotility related to diabetes is incompletely understood, but important 'downstream' consequences are related to alterations in the gut microbiota [37]. Dysfunction within the small bowel is well described in patients with long-standing diabetes, especially in those with coexisting gastroparesis [38]. Camilleri et al reported that small intestinal motility was abnormal in approximately $80 \%$ of patients with long-standing diabetes and concomitant delayed GET [39]. This finding leads to the hypothesis that the dysfunction of intestinal motility may predispose to SIBO. Hitherto there has, however, been a paucity of data on whether such SIBO influences colonic sensorimotor function. We, and others, have previously reported that measuring the $\mathrm{pH}$ drop across the ICJ may represent a non-invasive biomarker of the quantity of bacterial fermentation in the caecum $[17,29]$. Short-chain fatty acids are an end-product of bacterial fermentation and are the major contributor to acidity in this region of the gastrointestinal tract. In patients with functional gastrointestinal disorders, excessive fermentation in the caecum, as putatively indexed by a lower $\mathrm{pH}$ across the ICJ, is associated with a reduction in caecal and colonic contractility, as well as symptoms such as bloating and distension [40]. Considering that up to $60 \%$ of patients with type 1 diabetes report constipation, this could potentially represent the clinical sequelae of such alterations in the microbiota [38]. The mechanism(s) by which alterations in microbiota, or indeed shortchain fatty acids, contribute to alterations in colonic motility remain incompletely understood. It has been proposed that short-chain fatty acids may act as an intraluminal stimulus, thereby modifying motility patterns within the hindgut [29]. Whether alterations in microbiota are a cause, or indeed an effect, of changes in motility remain to be fully determined. Nevertheless, modulation of gut microbiota in animal models using antibiotics has been demonstrated to improve both insulin signalling and glucose tolerance [41]. Such heightened fermentation in the caecum is, however, a plausible potential therapeutic target by using interventions to alter the composition of the microbiota, which might lead to improvements in colonic motility; this warrants further objective evaluation.

Despite our findings, our study was subject to a number of limitations. First, although we used validated questionnaires to evaluate gastrointestinal symptoms, the interpretation of such relatively crude tools can be influenced by a degree of recall bias, particularly as we did not record symptoms during the WMC test. Second, although the patient and healthy cohorts were well matched from an age perspective, there were more men in the patient cohort; however, we used the previously defined sex-specific transit times in our analysis [15]. Third, the administration of a standardised meal during testing, in this 
case the SmartBar (Medtronic, Minneapolis, MN, USA), may not represent a typical meal for a patient with diabetes, potentially influencing motility patterns. In addition, we only measured baseline blood glucose rather than using continuous monitoring. Interestingly, we did not observe any associations between baseline blood glucose and segmental or pan-enteric transit times, in contrast to previous studies which have demonstrated that hyperglycaemia slows gastric emptying, while hypoglycaemia profoundly accelerates it [42]. For instance, Russo et al reported that gastric emptying of both solids and liquids is faster during insulin-induced hypoglycaemia in patients with type 1 diabetes, suggesting that gastric motility is an important compensatory mechanism in this context. Fifth, the generalisability of these findings to patients with other types of diabetes, in the absence of neuropathy, remains uncertain. A further point is that the WMC is an indigestible capsule that may not in itself accurately represent transit times and motility under normal physiological conditions. Finally, an inherent limitation of the study is the potential confounding factor of the presence of SIBO and/or lactose intolerance, which we did not objectively test for. In diabetes, impaired intestinal motility can be complicated by SIBO [43, 44]. However, the prevalence of SIBO in patients with diabetes is unclear as there are marked differences dependent on a number of factors including the presence or absence of symptoms, the presence or absence of concomitant DAN and type 1 vs type 2 diabetes $[45,46]$. Furthermore, breath-testing for SIBO is also subject to a number of limitations including lack of sensitivity and specificity, choice of carbohydrate substrate used in the test, measurement of other products of fermentation beyond hydrogen and lack of international standardisation in the interpretation of test results $[47,48]$. With respect to lactose malabsorption, although we did not specifically test for lactose intolerance, none of the participants reported in their medical history features consistent with this, such as abdominal pain and distension, borborygmi, flatus and/or diarrhoea induced by lactose in dairy products. Self-reported symptoms perform only modestly in excluding lactose intolerance, although the prevalence of lactase persistency, which therefore makes lactose intolerance less likely, is relatively high given the demographics of the study population [49]. However, Rana et al have proposed that there are a number of downstream effects of lactose malabsorption in patients with diabetes, such as lower levels of serum calcium and the presence of osteoporosis, which were not present in our patient cohort, suggesting that this was not a significant confounding factor in our study [50].

In summary, although recent studies have focused on gastroparesis and symptoms in type 1 diabetes, this study shows a pan-enteric prolongation of transit times prior to the potential development of bothersome symptoms. Thus, treatments that aim to arrest the development and worsening of dysmotility need to be developed. Furthermore, we have demonstrated that patients with prolonged gastrointestinal transit and heightened $\mathrm{pH}$ change across the ICJ potentially do not report severe gastrointestinal symptoms. Therefore, it is likely that the current burden of disease is probably underrecognised and underappreciated by clinicians. In conclusion, our finding of delayed transit times and heightened fermentation highlights the need to proactively encourage patients to modify the lifestyle factors such as daily water intake, dietary aspects and physical exercise in order to potentially minimise the consequences of diabetes-induced gut dysmotility. Moreover, recognising patterns of gastrointestinal tract transit abnormalities and alterations in the gastrointestinal $\mathrm{pH}$ profile may contribute to our understanding of the pathophysiological mechanisms behind these disorders, which could ultimately lead to the development of new therapies.

Acknowledgements The abstract from a proportion of the entire dataset was presented as an oral presentation at Digestive Disease Week 2016, San Diego, CA, USA.

Data availability The datasets generated and/or analysed during the current study are available from the corresponding author on request.

Funding The study was supported by the Novo Nordisk Scandinavia AS, Empowering Industry and Research EIR Northern Jutland and the Innovation Fund Denmark, Individuals, Disease and Society, Copenhagen, Denmark (Grant no. 10-092786). CB received funding from the Talent Programme, Aalborg University. ADF was supported by the Danish Diabetes Academy funded by the Novo Nordisk Foundation and the Research and Development Department, University Hospitals of North Midlands.

Duality of interest The authors declare that there is no duality of interest associated with this manuscript.

Contributions statement $\mathrm{BB}, \mathrm{AMD}, \mathrm{CB}$ were responsible for the original idea and study design. Data collection and analysis were carried out by ADF, AGP, PEJ, JSK, SDM, SMS and CB. ADF and CB drafted the manuscript. All authors contributed to the literature search and preparation of the manuscript and critical revisions therein in terms of important intellectual content, and approved the final manuscript. CB is the guarantor of this work.

\section{References}

1. Tesfaye S, Boulton AJ, Dyck PJ et al (2010) Diabetic neuropathies: update on definitions, diagnostic criteria, estimation of severity, and treatments. Diabetes Care 33:2285-2293

2. Bansal V, Kalita J, Misra UK (2006) Diabetic neuropathy. Postgrad Med J 82:95-100

3. Brock C, Softeland E, Gunterberg Vet al (2013) Diabetic autonomic neuropathy affects symptom generation and brain-gut axis. Diabetes Care 36:3698-3705 
4. Krishnan B, Babu S, Walker J, Walker AB, Pappachan JM (2013) Gastrointestinal complications of diabetes mellitus. World $\mathrm{J}$ Diabetes 4:51-63

5. Locke GR 3rd (1995) Epidemiology of gastrointestinal complications of diabetes mellitus. Eur J Gastroenterol Hepatol 7:711-716

6. Grover M, Farrugia G, Lurken MS et al (2011) Cellular changes in diabetic and idiopathic gastroparesis. Gastroenterology 140(15751585), e 1578

7. Azpiroz F, Malagelada C (2016) Diabetic neuropathy in the gut: pathogenesis and diagnosis. Diabetologia 59:404-408

8. Camilleri M, Bharucha AE, Farrugia G (2011) Epidemiology, mechanisms, and management of diabetic gastroparesis. Clin Gastroenterol Hepatol 9:5-12, quiz e17

9. Kempler P, Varkonyi T, Korei AE, Horvath VJ (2016) Gastrointestinal autonomic neuropathy in diabetes: the unattended borderline between diabetology and gastroenterology. Diabetologia 59:401-403

10. Choung RS, Locke GR 3rd, Schleck CD, Zinsmeister AR, Melton LJ 3rd, Talley NJ (2012) Risk of gastroparesis in subjects with type 1 and 2 diabetes in the general population. Am J Gastroenterol 107: 82-88

11. Wang YR, Fisher RS, Parkman HP (2008) Gastroparesis-related hospitalizations in the United States: trends, characteristics, and outcomes, 1995-2004. Am J Gastroenterol 103:313-322

12. Boronikolos GC, Menge BA, Schenker N et al (2015) Upper gastrointestinal motility and symptoms in individuals with diabetes, prediabetes and normal glucose tolerance. Diabetologia 58:1175-1182

13. Arora Z, Parungao JM, Lopez R, Heinlein C, Santisi J, Birgisson S (2015) Clinical utility of wireless motility capsule in patients with suspected multiregional gastrointestinal dysmotility. Dig Dis Sci 60: 1350-1357

14. Tornblom H (2016) Treatment of gastrointestinal autonomic neuropathy. Diabetologia 59:409-413

15. Wang YT, Mohammed SD, Farmer AD et al (2015) Regional gastrointestinal transit and $\mathrm{pH}$ studied in 215 healthy volunteers using the wireless motility capsule: influence of age, gender, study country and testing protocol. Aliment Pharmacol Ther 42:761-772

16. Cesario V, Di Rienzo TA, Campanale M et al (2014) Methane intestinal production and poor metabolic control in type I diabetes complicated by autonomic neuropathy. Minerva Endocrinol 39: 201-207

17. Ringel-Kulka T, Choi CH, Temas D et al (2015) Altered colonic bacterial fermentation as a potential pathophysiological factor in irritable bowel syndrome. Am J Gastroenterol 110:1339-1346

18. Farmer AD, Scott SM, Hobson AR (2013) Gastrointestinal motility revisited: the wireless motility capsule. United Eur Gastroenterol J 1:413-421

19. Sarosiek I, Selover KH, Katz LA et al (2010) The assessment of regional gut transit times in healthy controls and patients with gastroparesis using wireless motility technology. Aliment Pharmacol Ther 31:313-322

20. Thakur D, Paudel BH, Bajaj BK, Jha CB (2010) Nerve conduction study in healthy individuals: a gender based study. Health Renaiss 8:169-175

21. Umetani K, Singer DH, McCraty R, Atkinson M (1998) Twenty-four hour time domain heart rate variability and heart rate: relations to age and gender over nine decades. J Am Coll Cardiol 31:593-601

22. Merio R, Festa A, Bergmann $\mathrm{H}$ et al (1997) Slow gastric emptying in type I diabetes: relation to autonomic and peripheral neuropathy, blood glucose, and glycemic control. Diabetes Care 20:419-423

23. Ewing DJ, Burt AA, Williams IR, Campbell IW, Clarke BF (1976) Peripheral motor nerve function in diabetic autonomic neuropathy. J Neurol Neurosurg Psychiatry 39:453-460

24. Yarandi SS, Srinivasan S (2014) Diabetic gastrointestinal motility disorders and the role of enteric nervous system: current status and future directions. Neurogastroenterol Motil 26:611-624
25. Farrugia G (2015) Histologic changes in diabetic gastroparesis. Gastroenterol Clin N Am 44:31-38

26. Urbain JL, Vekemans MC, Bouillon R et al (1993) Characterization of gastric antral motility disturbances in diabetes using a scintigraphic technique. J Nucl Med 34:576-581

27. Sfarti C, Trifan A, Hutanasu C, Cojocariu C, Singeap AM, Stanciu C (2010) Prevalence of gastroparesis in type 1 diabetes mellitus and its relationship to dyspeptic symptoms. J Gastrointestin Liver Dis 19:279-284

28. Jones KL, Russo A, Stevens JE, Wishart JM, Berry MK, Horowitz M (2001) Predictors of delayed gastric emptying in diabetes. Diabetes Care 24:1264-1269

29. Farmer AD, Mohammed SD, Dukes GE, Scott SM, Hobson AR (2014) Caecal pH is a biomarker of excessive colonic fermentation. World J Gastroenterol 20:5000-5007

30. Frokjaer JB, Andersen LW, Brock C et al (2013) Altered brain microstructure assessed by diffusion tensor imaging in patients with diabetes and gastrointestinal symptoms. Diabetes Care 36:662-668

31. Lelic D, Brock C, Simren M et al (2014) The brain networks encoding visceral sensation in patients with gastrointestinal symptoms due to diabetic neuropathy. Neurogastroenterol Motil 26:46-58

32. Parkman HP, Hasler WL, Fisher RS, American Gastroenterological A (2004) American Gastroenterological Association technical review on the diagnosis and treatment of gastroparesis. Gastroenterology 127:1592-1622

33. Maqbool S, Parkman HP, Friedenberg FK (2009) Wireless capsule motility: comparison of the SmartPill GI monitoring system with scintigraphy for measuring whole gut transit. Dig Dis Sci 54:2167-2174

34. Kuo B, McCallum RW, Koch KL et al (2008) Comparison of gastric emptying of a nondigestible capsule to a radio-labelled meal in healthy and gastroparetic subjects. Aliment Pharmacol Ther 27:186-196

35. Feinle C, Kunz P, Boesiger P, Fried M, Schwizer W (1999) Scintigraphic validation of a magnetic resonance imaging method to study gastric emptying of a solid meal in humans. Gut 44:106-111

36. Pasricha PJ, Yates KP, Nguyen L et al (2015) Outcomes and factors associated with reduced symptoms in patients with gastroparesis. Gastroenterology 149(1762-1774), e1764

37. Boland BS, Edelman SV, Wolosin JD (2013) Gastrointestinal complications of diabetes. Endocrinol Metab Clin North Am 42:809-832

38. Phillips LK, Rayner CK, Jones KL, Horowitz M (2006) An update on autonomic neuropathy affecting the gastrointestinal tract. Curr Diab Rep 6:417-423

39. Camilleri M, Malagelada JR (1984) Abnormal intestinal motility in diabetics with the gastroparesis syndrome. Eur J Clin Invest 14: 420-427

40. Farmer AD, Hobson AR (2016) Intraluminal pH as a pathophysiological biomarker of fermentation in irritable bowel syndrome. Am J Gastroenterol 111:145

41. Carvalho BM, Guadagnini D, Tsukumo DM et al (2012) Modulation of gut microbiota by antibiotics improves insulin signalling in high-fat fed mice. Diabetologia 55:2823-2834

42. Phillips LK, Deane AM, Jones KL, Rayner CK, Horowitz M (2015) Gastric emptying and glycaemia in health and diabetes mellitus. Nat Rev Endocrinol 11:112-128

43. Roza AM, Edmiston CE Jr, Frantzides C et al (1992) Untreated diabetes mellitus promotes intestinal microbial overgrowth. Am J Surg $163: 417-421$

44. Virally-Monod M, Tielmans D, Kevorkian JP et al (1998) Chronic diarrhoea and diabetes mellitus: prevalence of small intestinal bacterial overgrowth. Diabetes Metab 24:530-536

45. Cuoco L, Montalto M, Jorizzo RA et al (2002) Eradication of small intestinal bacterial overgrowth and oro-cecal transit in diabetics. Hepatogastroenterology 49:1582-1586

46. Zietz B, Lock G, Straub RH, Braun B, Scholmerich J, Palitzsch KD (2000) Small-bowel bacterial overgrowth in diabetic subjects is 
associated with cardiovascular autonomic neuropathy. Diabetes Care 23:1200-1201

47. Corazza GR, Menozzi MG, Strocchi A et al (1990) The diagnosis of small bowel bacterial overgrowth. Reliability of jejunal culture and inadequacy of breath hydrogen testing. Gastroenterology 98:302-309

48. Siddiqui I, Ahmed S, Abid S (2016) Update on diagnostic value of breath test in gastrointestinal and liver diseases. World J Gastrointest Pathophysiol 7:256-265
49. Jellema P, Schellevis FG, van der Windt DA, Kneepkens CM, van der Horst HE (2010) Lactose malabsorption and intolerance: a systematic review on the diagnostic value of gastrointestinal symptoms and self-reported milk intolerance. QJM 103:555-572

50. Rana S, Morya RK, Malik A, Bhadada SK, Sachdeva N, Sharma G (2016) A relationship between vitamin D, parathyroid hormone, calcium levels and lactose intolerance in type 2 diabetic patients and healthy subjects. Clin Chim Acta 462:174-177 